\title{
Cléopatre ou la mise en scene du changement propose
}

André A. Lafrance

\section{(2) OpenEdition}

1 Journals

Édition électronique

URL : http://journals.openedition.org/communicationorganisation/1986

DOI : 10.4000/communicationorganisation. 1986

ISSN : $1775-3546$

Éditeur

Presses universitaires de Bordeaux

Édition imprimée

Date de publication : 1 novembre 1997

ISSN : 1168-5549

Référence électronique

André A. Lafrance, «Cléopatre ou la mise en scene du changement propose », Communication et organisation [En ligne], 12 | 1997, mis en ligne le 19 décembre 2012, consulté le 19 avril 2019. URL : http://journals.openedition.org/communicationorganisation/1986 ; DOI : 10.4000/ communicationorganisation. 1986

Ce document a été généré automatiquement le 19 avril 2019

(c) Presses universitaires de Bordeaux 


\title{
Cléopatre ou la mise en scene du changement propose
}

\author{
André A. Lafrance
}

1 On nous a demandé, dans ce colloque, de nous intéresser au rôle de l'induction dans la communication. Nous aurions pu aller chercher des analogies du côté de l'induction électrique ou de l'induction méthodologique. Mais nous travaillons depuis déjà un certain temps sur une autre forme d'induction, celle qui mène à la transe hypnotique. Nous nous proposons de démontrer que l'étude de cette forme d'induction peut éclairer une façon dont les acteurs de l'échange communicationnel peuvent en occulter une étape importante que nous allons appeler « la transaction potentiaire ».

2 Notre démonstration portera sur l'action de communication qui vise le changement organisationnel. Ce changement, qu'il soit imposé ou dialogique, implique essentiellement la communication d'une nouvelle vision de l'organisation ou des relations entre ses composantes. Cette nouvelle vision doit d'abord être annoncée avant d'être expliquée, négociée ou imposée. Or cette annonce n'est pas limitée au contenu apparent du changement. Elle présente aussi, dans sa mise en scène, l'illustration du pouvoir dont l'acteur initiant le changement croit pouvoir disposer dans l'explication, la négociation ou l'imposition de ce dernier.

3 Étant à la fois historien et communicologue, nous allons présenter notre démarche à travers celle d'un personnage historique, Cléopâtre. Les études de cas historiques ont l'avantage d'offrir des objets clos dans leur développement événementiel. Ils sont documentés par des observateurs qui ne peuvent être accusés de partialité envers l'interprétation communicationnelle en voie d'élaboration, même s'ils peuvent l'être envers l'interprétation politique qui, contrairement à la première, était de leur temps et de leur intérêt. Lorsqu'ils font partie de la mémoire collective (enrichie par l'imaginaire des générations), de tels cas peuvent contribuer à l'élaboration d'un modèle, à condition qu'on ne prétende pas en tirer, aussi, la validation.

4 Nous allons retenir le cas de cléopâtre proposant un changement d'alliance à deux généraux romains: Jules César et Marc Antoine. La mise en scène est différente; le pouvoir dont elle dispose n'est pas le même dans les deux situations. Elle avait le talent de 
ses ancêtres : elle savait créer une symbiose entre les codes grecs et les rituels égyptiens. Elle répondait ainsi aux attentes des hommes forts romains, acteurs soumis aux fantasmes gréco-orientaux de leurs concitoyens romains. Octave-Auguste, l'adversaire de Marc Antoine, ne s'est d'ailleurs pas trompé sur la signification de ces mises en scène puisqu'il les a dénoncées comme preuves d'une soumission à des pouvoirs étrangers à l'Empire.

\section{Décrivons les acteurs de la première mise en scène}

D'abord Cléopâtre. La dynastie des Lagides ont dominé l'Égypte de 304 avant J.C. jusqu'en 30 après J.C., plus de trois cents ans après la mort d'Alexandre qui avait fait de sa capitale, Alexandrie, une charnière entre l'Occident et l'Orient méditerranéens. Ces pharaons grecs ont su faire la symbiose entre les deux cultures, grecque et égyptienne. Le célèbre phare d'Alexandrie n'était pas seulement un guide pour les marins. C'était aussi aussi un symbole de son rayonnement culturel, comme en témoignent la bibliothèque et le gymnase où les plus fins esprits de l'époque se retrouvent.

6 Les Lagides (du nom de Lagos, père du premier Ptolémée) ont assumé les rites et les traditions pharaoniques. Le Pharaon Ptolémée XIII a épousé sa sœur Cléopâtre. Ils devraient normalement régner ensemble. Mais - cela est aussi malheureusement dans la tradition des pharaons - le frère et la sœur sont en guerre ouverte. Conseillé par deux intriguants, le jeune Ptolémée a exilé sa sœur. Celle-ci a monté une armée qui attend d'affronter celle de son frère qui occupe, seul, le trône et la capitale.

7 Dans l'empire romain, c'est aussi la guerre civile. Jules César vient de battre - contre toutes les prédictions - Pompée dans la bataille navale de Pharsale. Pompée est en fuite. César le poursuit. En 48 avant J.C., les deux adversaires vont débarquer à Alexandrie. Pompée qui a contribué à remettre sur le trône le père de Ptolémée alors qu'il en avait été chassé par son peuple, espère y trouver un allié. Mais, craignant que cette association ne pousse le vainqueur, Jules César, à mettre fin à l'indépendance - toute relative - de l'Égypte, le jeune Pharaon croit pouvoir s'assurer sa reconnaissance en faisant assassiner Pompée. Quand Jules César débarque, on lui offre la tête de son adversaire. Celui-ci ne semble pas particulièrement heureux de ce dénouement. Les fidèles de Pompée forment encore un groupe important dans le reste de l'empire. D'autres batailles attendent César. Il s'est lancé à la poursuite de Pompée avec une toute petite partie de ses troupes. Il serait bien heureux de mettre la main sur les ressources de l'Égypte.

8 À cette époque, les régions les plus prospères et les plus intéressantes stratégiquement étaient celles de l'Orient méditerranéen. Elles couvraient d'abord un immense territoire empreint d'hellénisme, et les plus grandes villes du monde antique comme Ephèse ou Pergame, servaient de relais à la diffusion de la culture grecque. Mais l'Orient, c'était surtout le royaume d'Égypte... Le royaume de Cléopâtre était l'un des pays les plus riches de l'époque, le grenier à blé de la Méditerranée et disposait d'une flotte considérable. (Calvat $1995: 45)^{1}$.

Mais comment César pourrait-il contrôler ces ressources alors qu'il n'a aucune confiance en ce jeune Pharaon? Il s'enferme dans le somptueux palais d'Alexandrie. Les conseillers de Ptolémée font tout pour susciter le ressentiment du peuple d'Alexandrie contre ces nouveaux occupants. 
10 Cléopâtre doit convaincre César de changer sa vision des choses. Elle doit lui montrer qu'elle ferait une meilleure alliée que son frère.

\section{Les arguments hypothétiques}

11 L'action de communication de Cléopâtre a essentiellement pour objet de changer l'autre. Or l'autre ne saurait accepter le changement sans chercher à évaluer les risques qu'il comporte pour lui. Dans ces deux partenaires de la communication, il y a Cléopâtre qui a déjà jugé le changement comme positif, pour elle et pour son trône, et qui essaie d'obtenir la participation de l'autre à son implantation. Et il y César qui n'a pas encore jugé le changement proposé parce qu'il vient d'en prendre connaissance.

On suppose que la communication entre les deux partenaires portera sur la valeur du changement. Le premier essaiera de faire partager au deuxième les avantages qu'il y trouve. Pour être réalisé par le récepteur, ce changement doit être évalué par ce dernier quant aux avantages attendus par rapport aux efforts perçus comme nécessaires pour obtenir ces avantages.

13 Cléopâtre est convaincue qu'une alliance entre César et elle-même pourra mieux servir les intérêts du général romain... et les siens.

14 Nous transformons en fraction d'adoption la fraction de sélection proposée par Rivers et Schramm $(1957)^{2}$ pour décrire le processus de choix entre plusieurs offres médiatiques. Il y a, dans l'adoption du changement, un mécanisme semblable :

15 La force motrice du gain attendu sera divisée par l'effort perçu comme nécessaire pour obtenir ce gain. $(G / E)$

16 Ces gains et ces efforts sont annoncés et énoncés par l'émetteur. Ils composent les arguments les plus apparents de son message. Cléopâtre peut mettre au service de César les ressources de l'Égypte. Plus intelligente et plus habile que son frère, elle n'a jamais trahi sa parole, comme son frère vient de le faire avec Pompée. Mais César risque alors de miser sur la mauvaise carte. Il doit faire l'effort de renverser la situation actuelle, au risque de se retrouver confronté à une guerre avec le peuple d'Alexandrie et l'armée de Ptolémée. Rien ne l'assure qu'il pourra, avec ses troupes réduites, remporter cette guerre...

17 ...que rendaient pour lui bien périlleuse le désavantage du temps et du lieu, un rigoureux hiver, l'activité d'un ennemi pourvu de tout, au sein de sa capitale, et son propre dénuement dans une lutte qu'il était loin de prévoir (Suétone, C.J. César XXX)

18 César pouvait craindre de faire le mauvais choix entre le frère et la sœur qui se disputaient le pouvoir alexandrin. Car, pour reprendre le mot de Gary Karrass : Il y a toujours une benne à ordures en route pour la décharge si nous voulons monter dedans. (Karrass $1986: 93)^{4}$

19 Comme il s'agit d'un changement à venir, ces arguments restent hypothétiques. Leur valeur dépend d'hypothèses élaborées par l'émetteur quant à l'évolution des vecteurs économiques (dans le sens large de l'économie des ressources) et politiques (dans le sens large des relations socio-techniques entre les acteurs) du contexte dans lequel se place le changement. Le récepteur porte un jugement non seulement sur les hypothèses, mais sur l'émetteur lui-même. Dans les hypothèses de l'émetteur, il y a une vision de l'organisation, telle qu'elle est et telle qu'elle devrait devenir après le changement 
proposé. Cette organisation est ici socio-politique comme elle pourrait être, dans d'autres cas, socio-économique ou socio-technique. Il doit s'ensuivre une acceptation ou un refus de ces visions de la part du récepteur. Les effets du changement ne peuvent être anticipés par le récepteur qu'en tablant sur la situation présente non pas de ce qui va changer, mais de ceux qui sont impliqués dans ce changement.

The key to understanding what might go on is really to understand what is going on. What I really do is try to figure out the present. (Naisbitt 85$)^{5}$

\section{Les arguments relationnels}

Les hypothèses de l'émetteur dépendent de sa capacité à les imposer au récepteur. Et pour le faire, il doit recourir à des arguments relationnels. Nous y retrouvons les deux composantes d'un message proposées par le groupe de Palo Alto : l'indice et l'ordre. Les arguments hypothétiques font partie du compte-rendu du message et la capacité de l'émetteur à imposer ses hypothèses, les arguments relationnels, de l'ordre précisant la façon dont les premiers arguments doivent être reçus.

Tout message (segment de communication) porte à la fois sur le contenu (le compte rendu - ou l'indice) et sur la relation (l'ordre); le premier niveau rend compte de la transmission d'une information sur des faits, des opinions, des sentiments, des expériences, etc. : le second définit la nature de la relation entre les informateurs. (Jackson $1981: 30)^{6}$

L'ordre permet de contextualiser le message. Cléopâtre doit changer la vision de César afin de l'amener à accepter ses arguments pour un changement d'alliance. Elle doit le convaincre que son évaluation du pouvoir de Ptolémée est erronée.

Nous allons appeler pouvoir cette capacité de l'émetteur à faire adopter ses hypothèses par le récepteur.

Ce pouvoir se conjugue sur une matrice dont un axe est sa nature et l'autre, sa source.

Plusieurs auteurs ont proposé une classification de ces pouvoirs. Par exemple, au sein de l'école structuraliste, Amitaï Etzioni (1968: 356-359) ${ }^{7}$ a proposé trois catégories : physique (coercitif), matériel (utilitaire) et symbolique (normatif). Nous proposerons, nous aussi, trois catégories. Nous sommes fort conscients que ce sujet pourrait entraîner de longues discussions. Mais, comme cette proposition n'est pas le but de notre communication, nous vous invitons à l'accepter pour les fins de notre démonstration.

Nous proposons que la nature du pouvoir pourrait être physique, matérielle ou d'expert. Cléopâtre n'a pas le pouvoir physique d'imposer le changement à César. Nous laissons de côté la complicité sensuelle qui pourrait s'être développée entre les deux personnages dans la suite de l'autre complicité, politique celle-là. Celle-ci ne relève pas du pouvoir physique qui suppose une coercition dont Cléopâtre ne possédait aucun des éléments. D'ailleurs le harcèlement sexuel dont on fait actuellement grand cas est condamnable par l'usage d'un autre pouvoir, le pouvoir matériel, ou déplorable par l'usage d'un prétendu pouvoir d'expert... qu'on tient absolument à démontrer !

César que ses adversaires décrivait comme «l'homme de toutes les femmes et la femme de tous les hommes " n'avait certainement pas besoin de Cléopâtre pour assouvir ses besoins en ce domaine. Mais elle disposait d'un pouvoir matériel par le contrôle éventuel des ressources de l'Égypte... si le changement annoncé se réalisait. 

comme une qualité de type univoque détenue par certains membres d'une organisation à l'exclusion des autres. Pour que les unités de communication donnent lieu aux séries d'interactions bi-directionnelles composant un ensemble communicationnel, il faut que tous les acteurs possèdent, d'une façon ou d'une autre, cet instrument déclencheur et intégrateur.

Ce pouvoir prend sa source dans le fait qu'il est attaché à la personne ou attaché à la tâche. Exilée de son trône, Cléopâtre ne pouvait prétendre détenir un pouvoir matériel attaché à la tâche de pharaon régnant. Mais elle avait un pouvoir attaché à la personne de par son héritage et de par la hardiesse de sa résistance aux attaques de son frère.

Pour être efficace, un pouvoir doit être reconnu non seulement comme détenu par l'émetteur, mais aussi comme détenu de façon plus importante par l'émetteur que par le récepteur.

\section{La transaction potentiaire}

L'émetteur annonce un pouvoir et le récepteur répond par un pouvoir d'une autre nature ou le même pouvoir de source différente. C'est ce que nous appelons la transaction potentiaire.

Le récepteur peut essayer de démontrer que, dans le pouvoir choisi, le niveau de l'émetteur n'est pas supérieur au sien et ne peut donc être considéré comme un instrument indiscutable pour forcer l'intégration du message. Pour cela, il n'aurait qu'à refuser de lui reconnaître le pouvoir choisi ou de prétendre le posséder à un niveau plus élevé (le « one up-manship » de la relation symétrique de Bateson).

Mais le récepteur peut aussi répondre par un autre pouvoir. À peut lancer l'échange avec un pouvoir matériel et $\mathrm{B}$ répondre avec un pouvoir d'expert. Par exemple, l'employé qui travaille sur une machine depuis plus de 3 ans croit avoir trouvé une façon de réduire les pertes entraînées par les déchets de découpage. Il propose sa solution au contremaître tablant sur le fait qu'il est, lui-même, celui qui connaît le mieux les opérations concernées (pouvoir d'expert attaché à la tâche). L'autre refuse cet argument (cet ordre de la communication) en se réfugiant derrière son pouvoir matériel ou son pouvoir d'expert attaché à la personne (un diplôme de maîtrise technique).

Comme dans une partie de cartes, l'émetteur sera appelé à changer de carte-pouvoir jusqu'à ce que l'autre lui reconnaisse un atout imbattable dans une relation complémentaire ou qu'il le force à déclarer forfait et à retirer le changement demandé.

L'action communicationnelle ne serait donc pas limitée à un seul échange d'offre et demande de pouvoir; en dehors des cas de contacts accidentels ou télégraphiques, il y aurait une série d'essais de part et d'autre jusqu'à ce que l'une des deux parties accepte une des propositions fournies par l'autre.

...dans la mesure où toute relation entre deux parties suppose échange et adaptation de l'une à l'autre et réciproquement, le pouvoir est inséparablement lié à la négociation: c'est une relation d'échange, donc de négociation, dans laquelle deux personnes au moins sont engagées (Crozier et Friedberg $1986: 352)^{8}$

38 Ces échanges pourraient d'ailleurs très bien se prêter aux méthodes d'analyse transactionnelle proposées par Éric Berne (1964) ${ }^{9}$. 

produit d'un processus structurant. En fait la communication devient alors un processus complexifiant. Contrairement aux arguments hypothétiques dont la validation est dépendante de la réalisation effective du changement, ces arguments relationnels sont immédiatement confrontés aux perceptions du moment. La transaction re-négocie la relation en actualisant diverses possibilités dans la nature et la source des pouvoirs de chacun des acteurs.

Analyser un texte ce n'est ni prétendre rendre compte du seul point de vue du sujet communiquant, ni être condamné à ne pouvoir rendre compte du seul point de vue du sujet interprétant; c'est rendre compte des possibles interprétatifs qui surgissent (ou se cristallisent) au point de rencontre des deux processus de production et d'interprétation, le sujet analysant étant un collecteurs de points de vue interprétatifs de façon à pouvoir en dégager, par comparaison, des constantes et des variables. (Charaudeau $1992: 57)^{10}$

\section{L'escalade}

41 Nous émettons l'hypothèse qu'il y a un ordre d'escalade dans l'utilisation des pouvoirs. Les trois niveaux de pouvoir (physique, matériel, d'expert) et les deux sources à l'intérieur de chaque niveau (attaché à la personne et attaché à la tâche) se situeraient sur deux échelles : l'une de contrainte et l'autre de satisfaction.

Dans la séquence de présentation (physique attaché à la tâche en premier), les pouvoirs imposeraient une contrainte décroissante. Si on a pu dire que, techniquement, la violence physique était un moyen beaucoup plus économique que le marché pour se procurer un bien, le pouvoir physique offre à l'émetteur une efficacité plutôt rassurante. Sa manipulation est relativement simple. Son application répond à un code bien rôdé. Sa perception par le récepteur est rapide.

43 Par contre, le pouvoir d'expert attaché à la personne, à l'autre bout de l'échelle, se situerait dans une zone d'incertitude particulièrement grande.

Dans la séquence inverse, le pouvoir d'expert attaché à la personne (l'incitation amicale) offrirait une plus grande satisfaction à l'utilisateur.

C'est la peur d'être seul qui le pousse à se faire valoir, à mériter une place. C'est la peur d'être seul qui lui fait éviter les conflits et le contraint à séduire. (Pelletier 1981) ${ }^{11}$

46 À l'autre bout du continuum, le pouvoir physique attaché à la tâche (l'exigence contraignante) serait, en dehors des cas pathologiques, la reconnaissance de l'incapacité de faire reconnaître la possession de pouvoirs liés à la personne de l'émetteur.

Il y aurait donc une tendance naturelle à entamer un échange par un pouvoir d'expert attaché à la personne et procéder à l'escalade jusqu'à l'obtention d'une validation. Sachant qu'il est plus valorisant pour l'émetteur d'éviter l'escalade, le récepteur joue le jeu ou prend le risque de faire monter les enjeux. D'ailleurs on pourrait se demander si l'organisation ne balise pas, par ses pratiques, le cheminement de la transaction.

Organizing is the grammar by which the vocabulary of elements in an organization is made meaningful (Weick 1969 : 62) ${ }^{12}$. 


\section{La transe-action potentiaire}

Pour éviter l'escalade et la remise en question des pouvoirs respectifs, l'émetteur cherche les moyens de placer le récepteur dans un état de disponibilité à la communication et au changement demandé. L'idéal, pour lui, serait que cet état de disponibilité soit tel que le récepteur accepte, sans prendre ni le temps ni le risque de mettre en cause, non seulement les arguments hypothétiques à cause de l'argument relationnel (le pouvoir énoncé), mais aussi l'argument relationnel lui-même.

On pourrait comparer cet état, apparamment idéal pour l'émetteur, à la transe hypnotique. Celle-ci a justement pour but de concentrer l'attention du sujet sur les suggestions offertes par l'hypnotiseur et d'obtenir ainsi une plus grande disponibilité à les accepter. Nous allons voir que cette analogie va plus loin qu'une simple coïncidence d'intentions.

L'hypnose est, elle aussi, une transaction entre deux personnes, l'une jouant le rôle de l'hypnotiseur (l'émetteur) et l'autre celui du sujet (le récepteur). La transe ou l'état qui en résulte, a fait l'objet de nombreuses discussions dans les milieux spécialisés.

Ce lien entre la communication et l'hypnose a été traité de diverses façons par de nombreux auteurs. Dans son ouvrage Trancework, Michael D. Yapko, parle de communication thérapeutique qui amène une personne en détresse à penser ou à se comporter différemment. Par rapport à l'hypnose traditionnelle, cette communication mettrait l'accent moins sur le rituel et le niveau de transe, et plus sur l'utilisation de mots et de gestes particuliers. Ainsi toute communication pourrait avoir des qualités hypnotiques sans être une hypnose formelle. $(1990: 11-12)^{13}$

33 Pour certains, la transe est un phénomène fort commun. On la retrouve chez le lecteur et le téléspectateur aussi bien que chez le mystique. Une transe est toujours créée par une limitation de l'attention. Cette limitation est provoquée par une répétition de sons, de bruits, de mots ou d'images qui surchargent intellectuellement ou émotivement le cerveau. Celui-ci n'a plus d'autre choix que de fuir dans une transe qui le libère de cette surcharge. Il s'ensuit une atrophie de certaines fonctions mentales comme le jugement, la volonté, la conscience du corps, la continuité du temps, la mémoire.

Une transe hypnotique serait un type spécial de transe dans laquelle la boucle d'attention est connectée à des stimulus extérieurs au cerveau. L'hypnotiseur se sert du fait que la transe désactive des fonctions mentales pour introduire des suggestions. Ces suggestions deviennent des stimulus qui ne sauraient pas normalement accepter par le jugement ou la volonté du sujet. L'hypnotiseur prend avantage de la disjonction cognitive pour influencer le comportement. Pour y arriver, il doit induire la transe. C'est ainsi que l'étude l'induction hypnotique pourrait nous aider à mieux comprendre le processus par lequel l'émetteur réussit à éviter la transaction potentiaire et les risques qu'il fait courir aux rôles que chacun des partenaires croit jouer dans l'organisation. En état de transe, le récepteur peut accepter le pouvoir énoncé sans prendre la responsabilité de le valider.

\section{La mise en scène}

L'indice (l'argument hypothétique) et l'ordre (l'argument relationnel) peuvent être en multiplex sur le même canal. Les mots choisis par l'émetteur pour décrire le changement peuvent comporter une description de la relation. Le concept d'indexicalité, emprunté à 
l'ethnométhodologie, nous incite à rechercher le sens particulier des mots dans leur contexte de production. suggestions directes, un sujet à ses volontés. Ce mythe propagé par l'hypnose de spectacle prend ses racines chez les premiers praticiens de l'ère moderne comme Mesmer et Charcot. Par des instructions claires, l'hypnotiseur entraîne le sujet dans un état modifié de conscience dont il profite pour réaliser les actions thérapeutiques, analgésiques ou 
spectaculaires auxquelles le sujet serait incapable de collaborer dans un état normal de conscience.

Il existe une pratique semblable de la transaction potentiaire. L'émetteur détient tous les pouvoirs. Ce postulat l'amène donc à nier toute possibilité de refus par le récepteur. Il lui suffit d'exprimer un pouvoir - n'importe lequel - pour que l'autre l'accepte. Qu'est-ce qui expliquerait cette efficacité magique?

La surcharge intellectuelle ou émotive qui entraîne la transe enlèverait au récepteur toute volonté d'amorcer une transaction. Il accepterait le pouvoir annoncé sans même tenter de l'évaluer à l'aune de sa perception de la situation. On retrouverait là, par exemples, une version édulcorée - et caricaturale - des différentes techniques de programmation neuro-linguistique qui ont fait les beaux jours de certaines formations magiques en management. Car le succès de l'implantation d'un changement ne dépendrait que de l'habileté de l'émetteur à imposer son pouvoir. Cette approche explique aussi, fort étrangement, un intérêt pour la culture d'entreprise qui introduirait dans le subconscient des membres de l'entreprise la primauté de différents pouvoirs attachés à la tâche des échelons hiérarchiques.

On comprend que l'efficacité à court terme d'une telle induction autoritaire nie les fondements de la distribution des pouvoirs. Si nous croyons que chaque individu a besoin de se voir confirmer certains pouvoirs pour jouer un rôle actif dans son milieu, cette négation ne pourra avoir que des effets pervers sur le fonctionnement de ce milieu.

On comprend aussi que cette approche ne puisse se contenter que d'une acceptation inconditionnelle, même passive, du récepteur. Dans l'hypnose, les praticiens de cette approche ne peuvent attribuer l'échec qu'à la résistance du sujet.

De même l'émetteur qui ne réussit pas à obtenir les changements requis du récepteur, considérera que ce dernier est le premier responsable de sa résistance. Il ne lui viendra jamais à l'idée que sa rigidité puisse y être pour quelque chose.

Mais il peut y avoir comme une version tronquée de cette induction. Car les récepteurs qui n'accepteraient pas le pouvoir suggéré pourraient le faire non par conviction, mais par habitude, par pudeur ou par prudence envers la transaction elle-même. Dans les programmes d'implantation du changement par la mise en transe des récepteurs, on assiste ainsi à la réduction du rôle des acteurs à celui de figurants. Revenons aux images de la transe. Elle peut-être un état modifié de conscience. Mais elle peut aussi relever d'un jeu de rôle dans lequel le récepteur choisit défaire semblant. Le sujet ne sent pas la transe. Mais il réalise les suggestions de l'hypnotiseur pour lui faire plaisir ou pour se faire plaisir.

72 Par habitude, les résultats de la transaction ont déjà été assumés, par le récepteur, lors de communications précédentes.

73 Par pudeur, le récepteur fait semblant d'ignorer la transaction au nom d'une culture égalitaire ou coopérative.

74 Par prudence, le récepteur évalue que l'enjeu du changement proposé ne justifie pas les risques d'une transaction dont les résultats sont imprévisibles.

75 L'obéissance automatique, inconditionnelle, permet d'éviter une réelle confrontation avec le supérieur, une confrontation qui pourrait déboucher en face à face sur une affirmation brutale de l'arbitraire le plus pur: "Tu le fais parce que je te dis de le faire ». Cette servilité automatique, qui permet d'éviter que les choses soient dites clairement, est vécue par tous comme une chose 
inévitable et dont il faut s'accommoder. Rares sont ceux qui y prennent réellement plaisir. (Feigen Fasteau : 1974) $)^{16}$

Supposons que, dans une transaction potentiaire, le récepteur juge qu'il ne peut accepter le postulat de l'émetteur. Il sait, par exemple, que le pouvoir d'expert de l'émetteur n'est pas supérieur au sien. Mais il n'ose pas le rejeter par crainte d'un autre pouvoir, par exemple matériel, auquel l'émetteur pourrait ensuite recourir. Il refuse de s'engager dans l'escalade. Il fera donc semblant d'accepter le pouvoir d'expert auquel il ne croit pas. Il est dans la même situation que le patient qui ne veut pas déplaire à son thérapeute.

Il peut aussi se livrer à ce jeu de rôle pour se faire plaisir. Voyons le spectateur qui se porte volontaire pour monter sur la scène et se livrer à des gestes inhabituels ou loufoques commandés par un hypnotiseur-comédien. Il peut alors laisser tomber toutes ses inhibitions et donner libre cours à un exhibitionnisme latent. Il se fait plaisir !

Le récepteur peut accepter le postulat de l'émetteur dans le seul but de ne pas avoir à assumer la responsabilité des actes qui en découleront. Cette acceptation passive n'implique aucun jugement sur le pouvoir utilisé. C'est tout simplement un refus de jouer l'engagement. Dans le premier cas, on acceptait pour ne pas avoir à refuser le postulat de l'émetteur. Ici, on accepte pour ne pas avoir à refuser le message. C'est ce qu'on retrouve dans un discours comme celui-ci: C'est lui le patron. Il est payé pour prendre des responsabilités. Moi, je fais ce qu 'on me dit de faire.

79 L'émetteur lui a fourni l'excuse pour se livrer à ses tendances naturelles de paresse, de manque de motivation ou de paranoïa. Il a pris sur lui la responsabilité du geste qu'il commande. Et le récepteur est trop heureux de se trouver ainsi libéré de son rôle d'acteur dans la situation en assumant le rôle que l'émetteur veut lui imposer dans la transaction.

Dans le contexte de l'éthique de l'hypnotiseur, on cite souvent le cas de cette jeune fille qui, dans la clinique de Charcot, est sortie de son état d'hypnose lorsque des étudiants lui ont donné l'instruction de se déshabiller. On y voit l'affirmation de l'égo reprenant du service lorsque l'instruction va à l'encontre de l'intégrité du sujet. Mais plusieurs auteurs ont démontré qu'une telle situation mettait en cause la confiance que le sujet mettait en l'émetteur. Il n'y avait pas, dans le cas cité, une très grande confiance. Si cela avait été le cas, le sujet en aurait peut-être déduit que le geste demandé n'était pas d'une nature dangereuse puisque quelqu'un de confiance demandait de le poser. Ou, à la limite, si le geste devait entraîner des conséquences graves, celui qui l'aurait commandé serait le seul à en porter la responsabilité. Le sujet pourrait ainsi se livrer à des gestes que sa conscience jugerait répréhensible, mais que son inconscient souhaiterait réaliser. (Christenson 1949: 37-54, Le Cron $1972: 49-50)^{17}$

\section{L'induction par le récepteur}

81 Des chercheurs ont porté leur attention sur l'autre partenaire, c'est-à-dire le sujet. On peut ainsi considérer la transe comme un état dont le sujet est le principal responsable. L'hypnotiseur prend alors le rôle de guide ou de facilitateur dans la démarche entreprise et dirigée par le sujet.

82 L'échec de l'induction serait uniquement dû à l'incapacité innée du sujet à entrer dans la transe. C'est pourquoi on a bâti des séries de tests expérimentaux permettant d'évaluer la suggestibilité du sujet. 
L'annulation de la transaction ne comporte donc pas, dans ce cas, d'effet négatif pour l'image potentiaire de l'émetteur. Sa volonté de ne pas recourir à l'escalade n'implique aucune conséquence à court terme, sinon le souhait d'éduquer ou de faire éduquer le récepteur pour qu'il en arrive à développer une susceptibilité plus grande à l'induction potentiaire.

Dans l'approche émetteur, ce dernier est actif et le récepteur, passif. Dans l'approche récepteur, c'est ce dernier qui est actif alors que l'émetteur reste passif après son action initiale. Une fois que l'émetteur a posé son postulat sur la table, il attend la réaction du récepteur. Il est au repos jusqu'à ce que le rejet de son postulat le force à se remettre en action.

5 Si la mise en scène de Cléopâtre devant César relevait plus de l'induction par l'émetteur, celle qu'elle a préparée pour Marc-Antoine laissait à ce dernier l'entière responsabilité d'entrer dans la transe proposée.

Contrairement à son prédécesseur, Marc-Antoine possédait tous les éléments du pouvoir matériel. Ayant partagé l'empire avec Octave, il s'était installé à Tarse en Cilicie pour préparer sa prochaine campagne contre les Parthes. Bien que Cléopâtre ait été confirmée par César dans son rôle de reine d'Égypte, elle savait que le Romain pouvait, à son bon vouloir, réquisitionner les ressources alimentaires et militaires d'un pays devenu, à toutes fins pratiques, une colonie. Elle avait besoin de sceller une alliance avec le nouveau maître de l'Orient afin de protéger un trône qui pouvait lui être enlevé du jour au lendemain. Elle savait bien qu'elle n'avait pas beaucoup d'amis à Rome. Les sénateurs n'avaient pas beaucoup de sympathie pour cette femme et les richesses (réelles ou supposées) de son royaume attisait la convoitise des plus ambitieux.

De quel pouvoir Cléopâtre pouvait se servir? La réponse lui apparut évidente. MarcAntoine, général romain ambitieux et épris de culture grecque, était fasciné par l'Orient. Plutôt nonchalant hors des champs de bataille, il rêvait de la sensualité dont s'inspiraient les rituels pharaoniques. Le raffinement dans la jouissance l'attirait beaucoup plus que la brutalité brouillonne des milieux romains. Il aspirait à incarner une nouvelle symbiose entre l'Occident et l'Orient. C'était là un fantasme (d'autres diraient « un projet») qui hantent les siècles. Sa démarche ne devait pas être très éloignée de celle que décrivait une philosophe contemporaine dans un ouvrage consacré à La connaissance de soi. (Davy 1966 :20)

Ainsi toute philosophie orientée vers la lumière se présente comme une démarche reliant l'Occident à l'Orient non point situés sur des plans géographiques, mais dans le sens symbolique de ces deux termes. Orient et Occident se trouvent à l'intérieur de l'être symbolisant l'aurore et le crépuscule. C'est en partant de son ombre, de son opacité, que l'homme à la recherche de la connaissance de soi se dirige vers sa propre réalité lumineuse, mû par le désir de la connaître et par conséquent de se connaitre. ${ }^{18}$

9 Mais pour justifier cette nouvelle version d'une domination romaine adaptée au milieu oriental, il avait besoin de développer et d'adopter une liturgie appropriée.

En cela, Cléopâtre avait un pouvoir d'expert attaché à la personne et à la tâche de reine d'Égypte... et d'ancienne complice de César. Ses ancêtres Lagides avaient habilement développé une idéologie capable de marier les héritages grecque et égyptien. Il lui suffisait simplement de l'annoncer par une mise en scène appropriée pour que MarcAntoine accède, par lui-même, à l'état de transe et accepte le pouvoir justifiant le changement souhaité par la reine. 
91 Sur le pont de son bateau, elle reposait à l'ombre des voiles pourpres du navire. Les avirons d'argent étaient maniés en cadence par les rameurs au son des cithares, des flutes et des chalumeaux. Cléopâtre était parée et peinte telles que l'étaient à cette époque les effigies d'Aphrodite. Des adolescents l'éventaient et de belles esclaves vêtues comme les Néréides et les Grâces, s'activaient au gouvernail et aux cordages. Des milliers de cierges d'encens semaient des effluves parfumées jusqu'aux rives du fleuve sur lequel la galère féerique voguait vers l'intérieur du pays. (Lissner $1957: 77)^{19}$

92 Cette approche récepteur suppose que l'induction se déroule toujours de la même façon. Chacun des deux acteurs joue son rôle sans vraiment tenir compte de l'interprétation de l'autre. L'hypnotiseur s'attache à suivre la procédure d'induction; le sujet à ne pas bloquer sa capacité naturelle à intégrer l'induction.

Marc-Antoine entre dans une transe qu'il a choisie et dans laquelle Cléopâtre lui sert de guide. Les observateurs de l'époque ne s'y sont pas trompés... même si les adversaires y trouvaient des arguments dépassant probablement la réalité voulue par le couple ainsi dénoncé. Quelque temps avant la bataille, Octave fit un discours devant ses troupes. Il nous est rapporté par Dion Cassius : Qui ne sangloterait en apprenant que des chevaliers et des sénateurs la flattent comme des eunuques? Qui ne se lamenterait en apprenant et en voyant qu'Antoine... a maintenant abandonné toutes les façons de vivre de nos pères, a épousé toutes les coutumes étrangères et barbares,... se prosterne au contraire devant cette femme comme devant une /sis ou une Sélénè, a pris enfin lui-même le nom d'Osiris et de Dionysos... Qu'on ne le considère plus comme Romain, mais comme Egyptien, ni ne l'appelle plus Antoine, mais d'un nom comme Sérapion! Qu'on ne considère plus qu'il a été un jour consul ou imperator, mais gymnasiarque! Car ces noms, il les a volontairement échangés avec les précédents et, après avoir rejeté tous les sujets de fierté de ses ancêtres, le voilà devenu joueur de cymbale de Canope... Il est impossible à qui vit dans un luxe de roi et une mollesse de femme de penser et d'agir en homme, puisque c'est une nécessité absolue qu'on s'assimile aux mœurs auxquelles on s'adonne. "Que pourrait-on craindre de lui ? Sa vigueur physique? lia dépassé la force de l'âge et il s'est efféminé. La force de son esprit ? Il agit comme une femme et vit en parfait débauché. (Dion Cassius) ${ }^{20}$

Pour réaliser le changement, on recourt alors à diverses techniques dites de motivation, de participation, de délégation ou de responsabilisation dont les programmes de réingénierie ou de qualité totale sont souvent de bons exemples. Ces techniques visent à aider les récepteurs à se mettre eux-mêmes en état de transe coopérative pour l'implantation du changement.

\section{L'induction transactionnelle}

L'approche émetteur ne tient aucun compte de la motivation du sujet. L'approche récepteur, de la sensibilité de l'hypnotiseur et de la relation qui s'établit entre ce dernier et le sujet. Or ces éléments font partie de l'approche d'un des plus célèbres praticiens de l'hypnose thérapeutique Milton H. Erickson : ${ }^{21}$

... l'hypnose devrait d'abord être le résultat d'une situation dans laquelle on développe, de façon constructive, des rapports interpersonnels et intrapersonnels qui serviront les objectifs, à la fois, de l'hypnotiseur et du sujet. On ne peut y arriver en suivant des procédures rigides, des méthodes figées ou en se contentant de ne satisfaire qu'un seul de ces objectifs. La complexité du comportement humain et ses motivations sous-jacentes exigent de connaître la multitude de facteurs existant dans toute situation impliquant deux personnes engagées dans une activité conjointe. (Erickson 1952) 22 

départ. La mise-en-scène serait établie sans possibilité de changement, autre que technique, en cours d'interprétation. Le contexte ainsi imposé à la communication ne serait pas appelé à évoluer. D'une conception où le sujet ne faisait que réagir aux actions de l'hypnotiseur, ou l'hypnotiseur à celles du sujet, ils deviennent véritablement coacteurs de la transe. On ne sait plus très bien qui est le sujet, et qui est l'hypnotiseur. Et même si cet état mettait le sujet en situation d'accepter plus facilement les suggestions de l'autre, c'est lui qui entraîne cet autre à entrer dans la transe du pouvoir attendu. En fait, le récepteur force l'émetteur à réévaluer son postulat relationnel. C'est, à proprement parler, une induction transactionnelle. L'émetteur est amené à remettre en question le pouvoir qu'il croyait détenir. Il peut même devoir imaginer qu'un autre pouvoir lui serait plus utile pour obtenir ou maintenir l'état de transe chez le récepteur. Il entre alors, lui même, dans un état de transe partagé où chacun des acteurs se concentre sur l'exploration du contexte organisationnel

\section{NOTES}

1. R. Calvat «Cléopâtre », Bulletin de l'Arelam, n XXXII, juillet 1995, pp. 43-57.

2. W. Rivers et W.Schramm, "The Impact of Mass Communication, in Responsibility in Mass Communication. New York. Harper \& Row, 1957.

3. Vie des douze Césars, Traduction de Théophile Beaudement, Paris, Flammanon, 1990

4. G. Karrass, Marché conclu: les stratégies de la négociation en affaires; Paris, Rivages/Les Echos, 1986. Trad. fr. de negociate to close, 1985

5. J Naisbitî, auteur du best-seller Megatrends dans Fortune 09/12/85

6. D.D. Jackson, dans P. Watzlawick et J.Weakland, Sur l'interaction. Palo Alto. 1965-197 », Paris, Le Seuil, 1981

7. A. Etzioni, The Act me Society: A Theory of Societal and Political Processo, New York, Free Press, 1968.

8. M. Crozier et E. Friedberg, L'acteur et le système. Paris, Le Seuil, 1977 réédition 1986

9. E. Berne. Games People Play, The psychology of human relationship. New York, Grove Press, 1964.

10. $P$ Charaudeau, Grammaire du sens et de l'expression. Paris, Hachette, 1992 
11. D. Pelletier, L'arc-en-soi : essai sur 1rs sentiments de privation et de plénitude, Paris, Montreal, $\mathrm{R}$ Laffont. Stanke, 1981.

12. K.E. Weick 1969 The Social Psychology of Organizing Reading, Addison-Westley Publishing Company, 1969

13. M.D.Yapco, Ph-D., Trancework : on introduction to the Pructtve of Clinical Hypnosis, NewYork, Brunner/Mazel, 1990.

14. A. Coulon. L'Êthnométhodologie, Presses Universitaires de France, coll. « Que sais-je ? », n² 2393, 1990

15. Traduction de Adrien F Vochelle, dans la version française de Oscar de Wertheimer, Cléopâtre. reine des rots. Pans, Payot, 1935

16. M. Feigen-Fasteau, The male machine. New York, Toronto, McGraw-Hill, 1974

17. J.A. Christenson Jr., «Dynamics in Hypnotic Induction,» In Psychiatry, 12 :37-54, 1949 Repris dans : L.M. Le Cron, Experimental Hypnosis, Secaucus, N.J., The Citadel Press,1972

18. M.M. Davy. La connaissance de soi, Paris, puf coll. « Le philosophe », 1966

19. Ivar Lissner, Les Césars, Paris, Corréa / Bûchet Chastel, 1957. Nous citons cet auteur à cause de sa description, et non pour recommander son interprétation historiques des intentions des personnages.

20. Dion Cassius, Histoire Romaine. L, 25-30, cité par R. Calbat, ibid.

21. il est intéressant de noter qu'Enckson eut des liens avec les membres de l'école de Palo Alto « Notre approche actuelle dérive pour une part directement de la thérapie familiale de base, et du travail d'Erickson, pour une autre part; mais elle en diffère aussi. ».

22. M.H. Erickson, The Collected Papers of Milton H. Erickson, éd. E.Rossi, New York, lrvington Publishers, vol. 1,1980, pp. 166-167

23. J.F. Chanlat, L'individu dans l'organisation : les dimensions oubliées, introduction. Québec, Les Presses de l'Université Laval 1985.

\section{AUTEUR}

\section{ANDRÉ A. LAFRANCE}

Université de Montréal, Québec, Département de communication 